\title{
The Euler equations in the columnar and rotational approximation: a model equation for asymptotic short capillary-gravity waves
}

\author{
Manna Miguel* ${ }^{\dagger}$ \\ Instituto de Física Teórica-UNESP, Rua Pamplona 145, 01405-900, São Paulo, Brasil \\ E-mail: 'manna@lpm. univ-montp2.fri
}

ABSTRACT: From a columnar approximation of the Euler equations of an incompressible fluid with surface tension, we derive in the short-wave approximation a new classical $1+1$ dimensional model equation for the motion of the surface. The side-band instability of the Stokes' waves is analyzed in terms of the physical parameters of the system.

\section{Introduction}

The nonlinear and dispersive propagation of surface waves in an ideal incompressible fluid of depth $h$ and density $\sigma$ under the action of gravity $g$ and surface tension $T$ is a classical

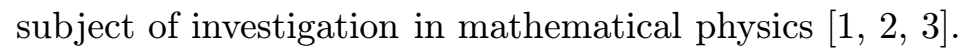

Surface wave are produced by the action of some external force on the (initial) plane free surface of the fluid in equilibrium. The motion of the surface will be propagated over the whole fluid in the form of waves which are called capillary-gravity waves. These waves are driven by a balance between the action of gravity and surface tension and the liquid inertia.

In this work we derive a new model equation which governs the asymptotic dynamics of a monochromatic short capillary-gravity wave

$$
u_{x t}+c u_{x x}=\frac{3 g}{2 c h}(1-3 \theta) u+\frac{3 h^{2}}{4 c} u_{x x} u_{x}^{2}-\frac{1}{4} u_{x}^{2}-\frac{1}{2} u_{x x} u
$$

where $u(x, t)$ is the fluid velocity on the surface ( $x$ and $t$ are space and time variables), subindices mean derivatives, $\theta=\left(T / \sigma h^{2} g\right)$ is the dimensionless Bond number and $c=$ $(3 T / \sigma h)^{1 / 2}$. Equation $\left(1 \overline{1} \overline{1}_{1}^{1}\right)$ has different regimes according to the values of $\theta$. In this work we consider only the case $\theta \neq 1 / 3$, for which $\left(\overline{1}_{1}^{1} \cdot \overline{1}_{i}^{\prime}\right)$ is a dispersive system.

${ }^{*}$ Speaker.

${ }^{\dagger}$ On leave of absence from Physique Mathématique et Théorique, CNRS-UMR5825, UM 2, France. 


\section{The surface water wave problem}

The dynamics of surface waves in an ideal fluid obeys nonlinear and dispersive equations. The whole analytical solution of the problem is imposible. To simplify the enormous complexity of the entire dynamics, multiscale asymptotic methods must be employed.

They produce asymptotic model equations (a.m.e) which describe only a small number of aspects. Most of them represent, for large $t$, balance between weak nonlinearities and linear dispersion. Nowadays a.m.e for monochromatic long-wave small perturbations of shallow dispersive fluids are very well known. They extend from the oldest Boussi-

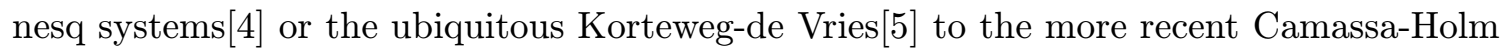
equation $[\bar{i}[\bar{i}]$ in which nonlinear dispersion replaces a linear one.

In contrast, hardly anything is known about a.m.e. for nonlinear and dispersive dynamics of monochromatic short-waves. For the most part short-waves have been studied in connection with modulation of short-wave trains $\left[\begin{array}{l}1 \overline{7} \\ 0\end{array}\right.$ of the causes of this situation arise.

\section{Asymptotic dynamics of short-waves}

When one speaks about short-waves one is referring to an underlying space scale to which the wave-lenght $l$ or the wave-number $k(k=2 \pi / l)$ of the linear plane wave solution $\exp i(k x-\omega t)$ must be compared. The frequency $\omega$ satisfies a dispersion relation $\omega=$ $W\left(k, p_{1}, p_{2}, \ldots\right)$ where $p_{i}$ are some physical parameters. In this case the unperturbed depth $h$ serves as a natural reference and we will consider $h \sim O(1)$ and $k \rightarrow \infty$ or $l \rightarrow 0$. Multiscale methods are strongly based on $W$ and on the associated notions of phase velocity $\left(v_{p}\right)$ and group velocity $\left(v_{g}\right)$, which define asymptotic variables essential to handle the nonlinear regime. These velocities have the sine qua non condition of being finite for the aspect we are presently looking at. In studies of monochromatic asymptotics, infinite dispersion $\left(v_{p} \rightarrow \infty\right)$, not only restrain us to define asymptotic variables but also cannot be compensated by weak nonlinerities. Therefore no a.m.e exist in this case. This is the situation in the classical linearization of the Euler equations with surface tension in the short-wave regime. The linear dispersion relation is $W=\left[k\left(g+T k^{2} / \sigma\right) \tanh (k h)\right]^{1 / 2}$ which yields in the short-wave limit $v_{p} \sim(T k / \sigma)^{1 / 2} \rightarrow \infty$.

\section{Asymptotic variables}

What is necesary to isolate the large time behavior of short-waves? We need to introduce a space variable $\zeta$ of order one for very small $x$ and a time variable $\tau$ of order one for very large $t$. Thus for $l=\epsilon \rightarrow 0, \zeta=x / \epsilon$ and $\tau=\epsilon t$ produce the description we are looking for. Variables $\zeta$ and $\tau$ must be meaningful at the linear level. Consequently, they must be compatible with and defined by the short-wave limit of $\mathrm{W}$. The more complete expression for $W(k)$ leading to the most general expressions for $\zeta$ and $\tau$ is a Laurent series with a simple pole for $k \rightarrow \infty$ so $W(k)=a k+b / k+c / k^{3}+\ldots$, where $a, b, c \ldots$ are real

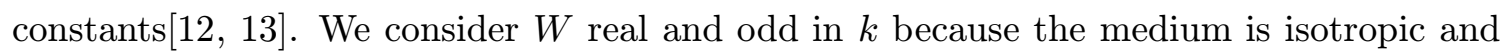


without dissipation. The solution found to avoid the transcendental dependence of $W$ in fonction of $k$ was to employ the Green-Nagdhi conditions of linearization.

\section{Asymptotic model equations for short capillary-gravity waves}

Irrotational fluid flow and the introduction of velocity potential are assumed in deriving $W$. They are not, however, the only hypothesis available for linearization. Using fundamental principles of continuum mechanics and Cosserat surface theories Green, Laws and

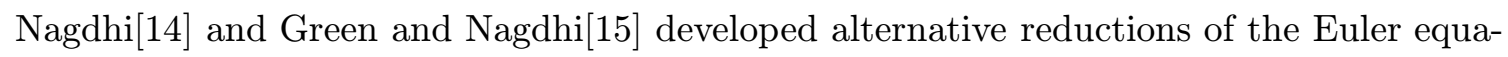
tions leading to model equations with polynomial dispersion relations. Non irrotational fluid flow moving in vertical columns were the two main hypothesis used.

Let the particles of the fluid medium be identified by a fixed rectangular Cartesian system of center $O$ and axes $(x, y, z)$ with $O z$ the upward vertical direction. We assume symmetry in $y$ and we will only consider a sheet of fluid in the $x z$ plane. This fluid sheet is moving in a domain with a rigid bottom at $z=0$ and an upper free surface at $z=S(x, t)$. The vector velocity is $\vec{v}=(u, w)$. The continuity equation $(\mathrm{C})$ is: $u_{x}+w_{z}=0$ and the Newton equations (in the flow domain) are: (N1) $\sigma\left(u_{t}+u u_{x}+w u_{z}\right)=-p_{x}^{*}$, and (N2) $\sigma\left(w_{t}+u w_{x}+w w_{z}\right)=-p_{z}^{*}-g \sigma$, where $p^{*}(x, z, t)$ is the pressure. The kinematic and dynamic boundary conditions are: $w(x, 0, t)=0$, and

$$
\begin{gathered}
S_{t}+u S_{x}-w=0, \quad z=S(x, t), \\
p^{*}=p_{0}-\frac{T S_{x x}}{\left(1+S_{x}^{2}\right)^{\frac{3}{2}}}, \quad z=S(x, t) .
\end{gathered}
$$

We assume that $u$ is independent of $z$. This is known as columnar-flow Ansatz. It is equivalent to consider the vertical component $w$ as a linear function of $z$. This enables us to satisfy exactly the equation of incompressibility and the boundary condition at the bed. Hence $u=u(x, t)$ and from (C) we have: $w=-z u_{x}$. The integration of (N1) in $z$ from 0 to $S(x, t)$ gives

$$
\sigma S\left(u_{t}+u u_{x}\right)=-p_{x}+T\left[\left(1+S_{x}^{2}\right)^{-\frac{1}{2}}\right]_{x} .
$$

where $p(x, t)=\int_{0}^{S(x, t)} p^{*}(x, z, t) d z-p_{0} S(x, t)$. Now we multiply (N2) by $z$ and integrate on $z$

$$
\sigma \frac{S^{3}}{3}\left(-u_{x t}-u u_{x x}+u_{x}^{2}\right)=p+\frac{T S S_{x x}}{\left[1+S_{x}^{2}\right]^{\frac{3}{2}}}-\frac{g \sigma S^{2}}{2} .
$$

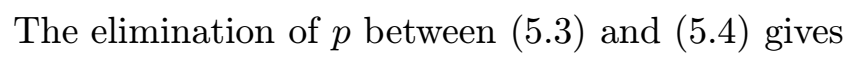

$$
\begin{aligned}
S\left(u_{t}+u u_{x}\right)= & \frac{1}{3}\left[S^{3}\left(u_{x t}+u u x x-u_{x}^{2}\right]_{x}-g S S_{x}+\right. \\
& \frac{T}{\sigma} S\left[\frac{S_{x x}}{\left(1+S_{x}^{2}\right)^{\frac{3}{2}}}\right]_{x} .
\end{aligned}
$$




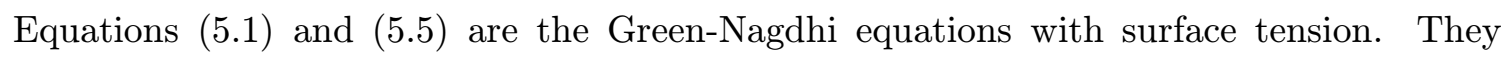
are a reduction of the Euler equations in a thin domain. In relation to shallow water theories with dispersion (Boussinesq type equations), Green-Nagdhi with surface tension incorporate finite dispersions in the long-wave and in the short-wave limits. The linear dispersion relation is in this case $W_{G N}^{2}=k^{2}\left[g h+(T h / \sigma) k^{2}\right] /\left[1+(h k)^{2} / 3\right]$ and the phase velocity for $k \rightarrow \infty$ reads $W_{G N} / k \sim(3 T / \sigma h)^{1 / 2}+O\left(1 / k^{2}\right)$

Now one can define asymptotic variables $\zeta=(1 / \epsilon)\left(x-v_{p} t\right)$ and $\tau=\epsilon t$ and with the expansion $u=\epsilon^{2}\left(u_{0}+\epsilon^{2} u_{2}+\ldots\right)$ and $S=h+\epsilon^{2}\left(W_{0}+\epsilon^{2} W_{2}+\ldots\right)$ nonlinear dynamics of small short capillary-gravity waves can be studied. Finally by perturbation in $\epsilon$ we obtain an equation for $u_{0}(\zeta, \tau)$ from which we obtain the main equation $\left(\overline{1}, \overline{1} \overline{1}_{1}\right)$ in laboratory variables.

\section{Benjamin-Feir instability}

In this section we study the resonant interaction occurring in a wave train (Stokes' wave train) with a narrow band of frequencies and wavelengths. Let us consider $u(x, t)$ as a plane wave. The nonlinears terms in (1) $\left(\begin{array}{l}1 \\ 1\end{array}\right)$ give rise to harmonics of the fundamental. Assume that a disturbance is present consisting of modes with sideband frequences and wavenumbers close to the fundamental. We can have interaction between harmonics and these sideband modes. This interaction is likely to produce a resonant phenomenon manifesting itself by the modulation of the plane wave solution. The exponencial growth in time of the modulation, originating from synchronous resonance between harmonics and sideband modes,

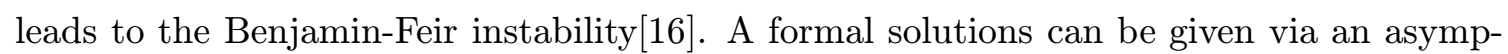
totic expansion conducing to the nonlinear Schodinger equation (NLS) [i] $\left.\overline{1}_{1}\right]$. The particular interest of NLS is the existence of a general and simple criterion enable to detect stability or unstability of the monochromatic wave train. Let us seek for a solution of (1) $1 \overline{1}$ i $)$ under the form of a Fourier expansion in harmonics of the fundamental exp $i(k x-\omega t)$ and where the Fourier components are developed in a Taylor serie in powers of a small parameter $\gamma$ mesuring the amplitude of the fundamental

$$
u=\sum_{l=-p}^{l=p} \sum_{p=1}^{\infty} \exp i l(k x-\omega t) \gamma^{p} u_{l}^{p}(\xi, \tau) .
$$

In ( $\left(\overline{6} \bar{b}_{1} \overline{1}_{\bar{l}}\right), u_{-l}^{p}=u_{l}^{* p}$ ("star" denotes complex conjugation) and $\xi$ and $\tau$ are slow variables introduced through the stretching $\xi=\gamma(x-v t)$ and $\tau=\gamma^{2} t$ and where $v$ will be determined as a solvability condition. The expansions $\left(\overline{6} \cdot \overline{1}_{i}\right)$ includes fast local oscillations through the dependence on the harmonics and slow variation (modulation) in amplitude taken into account by the $\xi, \tau$ dependence of $u_{l}^{p}$. Introducing now this expansion and the slow variables in (1) we may proceed to collect and solve different order $\gamma$ and $l$. We have with

$$
u_{1}^{1}=\psi(\xi, \tau), \quad a=\frac{3 g(1-3 \theta)}{2 c h}, \quad b=\frac{3 h^{2}}{4 c},
$$

the following conditions of solvability

$$
u_{0}^{1}=0, \quad \omega=k c+\frac{a}{k}, \quad v=2 c-\frac{\omega}{k}, \quad u_{0}^{2}=-\frac{k^{2}}{2 a}|\psi|^{2}, \quad u_{2}^{2}=\frac{k^{2}}{4 a} \psi^{2}, \quad u_{1}^{2}=\frac{i}{k} \psi_{\xi} .
$$


At order $\gamma=3, l=1$ (using $\left(\overline{6} \cdot \overline{3}^{i}\right)$ ) we obtain the NLS equation for $\psi(\xi, \tau)$ as solvability condition

$$
-i \psi_{\tau}-\frac{a}{k^{3}} \psi_{\xi \xi}+\left(\frac{1}{8 a}-b\right) k^{3} \psi|\psi|^{2}=0 .
$$

The nature of solutions of NLS depends drastically of the sign of the product between the coeficient of $\psi_{\xi \xi}$ and that of $\psi|\psi|^{2}$. In this case this product is positive for

$$
\theta<\frac{3}{10}
$$

and according to a well known stability criterion (see for exemple [i] is unstable, namely any slight deformation of the plane wave experiences an exponential growth.

\section{Conclusions}

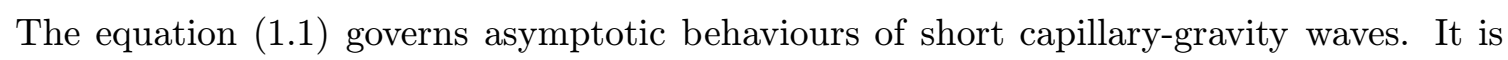
the short-waves analogous of the Korteweg-de Vries equation with surface tension which is derived in the long wave limit. Solutions of equation (1י1) must be strongly depended on the values of $\theta$, because this parameter governs dispersion. For exemple for $\theta=1 / 3$ equation

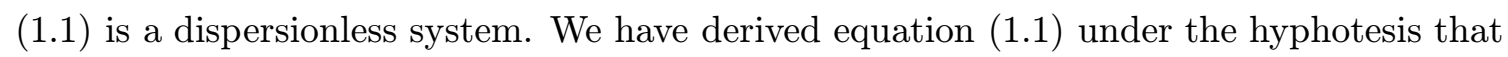
dissipative phenomena are small and the model represents asymptotic dynamics of linear dispersion and nonlinearity. However since dissipative phenomena take place at small scales viscosity must affect the asymptotic dynamics of short-waves. This is an important open problem. The analogous of equation (1, $\left.\overline{1}_{1}\right)$ in $(2+1)$ dimensions is the equation

$$
u_{x t}=\frac{3 g}{2 c h}(1-3 \theta) u+\frac{3 h^{2}}{4 c} u_{x x} u_{x}^{2}-\frac{1}{4} u_{x}^{2}-\frac{1}{2} u_{x x} u-\frac{c}{2} u_{y y}
$$

where $u(x, y, t)$ is the fluid velocity on the surface. It is the short-waves analogous of the Kadomtsev-Petviashvili equation with surface tension which is derived in the long

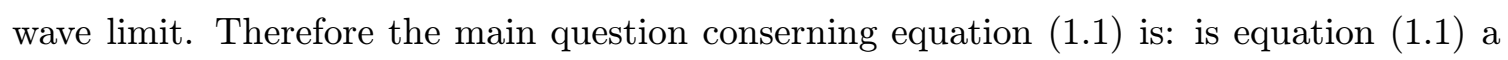
completely integrable system? A detailed study of all these open problems will be published elsewhere.

Aknowledgements The author wish to thank Professor R. Kraenkel for many helpful and stimulating discussions, the IFT for the hospitality and FAPESP for financial support.

\section{References}

[1] T. B. Benjamin, Q. Appl. Maths. 37, 231-234 (1982).

[2] J. K. Hunter and J.-M. Vanden-Broeck, J Fluid. Mech. 134, 205-219 (1983).

[3] F. Dias and C. Kharif, Annu. Rev. Fluid Mech. 31, 310-346 (1999).

[4] J. Boussinesq, Compte Rendus Acad. Sci. Paris 72, 755-759 (1871).

[5] D. J. Korteweg and G. de Vries, Phil. Mag. (5), 39, 422-443 (1895). 
[6] R. Camassa and D. D. Holm, Phys. Rev. Lett. 71, 1661-1664 (1993).

[7] M. S. Longuet-Higgins and R. W. Steward, J. Fluid. Mech. 8, 565-583 (1960) .

[8] S. J. Hogan, J. Fluid. Mech. 96, 417-445 (1980), J. Fluid. Mech. 110, 381-410 (1981).

[9] M. S. Longuet-Higgins, J. Fluid. Mech. 177, 293-306 (1987).

[10] C. C. Mei, The Applied Dynamics of Ocean Surface Waves (Adv. Series on Ocean Engineering vol 1) (Singapore: World Scientific) (1989).

[11] J. Zhang and W. Melville, J. Fluid. Mech. 214, 321-346 (1990), J. Fluid. Mech. 243, 52-72 (1992).

[12] M. A. Manna, Physica D 149, 231-235 (2001).

[13] M. A. Manna and A. Neveu, Inverse Problems, 17, 855-861 (2001).

[14] A. E. Green, F.R.S., N. Laws and P. M. Nagdhi, Proc. R. Soc. A 338, 43-55 (1974).

[15] A. E. Green, and P. M. Nagdhi, J. Fluid. Mech. 78, 237-246 (1976), Proc. R. Soc. A 347, 447-473 (1976).

[16] T. B. Benjamin and J. E. Feir, J. Fluid. Mech. 27, 417-430 (1967).

[17] J. T. Stuart and R. C. Di Prima, Proc. R. Soc. London A 362, 27 (1978).

[18] G. B. Whitham, Linear and Nonlinear Waves, (Wiley Interscience, New York, 1974). 